\title{
Energy Resources Management Enabled by Internet of Things Devices
}

\author{
${ }^{1,2}$ Luis Gomes, ${ }^{1,2}$ Pedro Faria, ${ }^{1,2}$ Francisco Silva, and ${ }^{2}$ Zita Vale \\ ${ }^{1} G E C A D$ - Research Group on Intelligent Engineering and Computing for Advanced Innovation and Development \\ ${ }^{2}$ Institute of Engineering - Polytechnic of Porto \\ Porto, Portugal \\ 0000-0002-8597-3383; 0000-0002-5982-8342; 0000-0003-4551-6732; 0000-0002-4560-9544
}

\begin{abstract}
The participation of small end-users in the smart grid brings benefits for the end-users and for the smart grid. This paper will treat end-users using communities involving energy sharing between private buildings (residential and commercial) and public buildings. The energy can be shared among end-users and the community can be managed centralized. The paper uses IoT devices to enable the active participation of end-users. The use of this type of devices is growing and more and more market available product are appearing. The remote control and monitor capabilities, provided by the normality of IoT devices, can and should be used in energy management systems as enablers. This paper uses IoT devices, located in end-users, to enable the participation of these player in the community. The paper will propose a smart energy community platform and show its results.
\end{abstract}

Keywords - community optimization, energy community, energy sharing, IoT devices for demand side management.

\section{INTRODUCTION}

The fast-growing market of Internet of Things (IoT) devices is changing the way people interact with, until now, unanimated objects. Worldwide, is expected that 216.9 million homes will have at least one IoT device by 2022 [1]. This market can be explored by manufacturers and by service providers - enabling new and improved functionalities that use IoT devices. The ability of IoT devices to remotely monitor and/or control resources, objects and/or the environment can be used in third-party applications to bring smartness and autonomous control to our buildings.

Currently in the market is possible to find some proprietary solutions that enable the integration of multiple brand IoT devices to build single intelligent systems. Voice assistant powered systems, such as Google Home, Amazon's Echo and Apple's HomePod, enables the integration of some IoT devices while providing voice assistant to users, as well as some automated mechanism for a more extended control. For open source solutions, is available Home Assistant and Mycroft that give the user higher liberty to change and configure his/her own solution. In the energy field, smart homes can highly benefit for the already available IoT devices, enabling the desired opportunity to control energy resources and monitor environmental contextual parameters. Therefore, IoT devices can be used for energy resources control, as seen in $[2,3]$. In $[4,5]$ are proposed two smart plugs for energy management applications. In [6] the Environmental Awareness Smart Plug (EnAPlug) is proposed, while in [7] a smart plug is proposed for consumption classification and profiling.
Smart homes enable the smart control over the electrical resources located in the home [8]. This enables energy management mechanism in houses and enhance the participation of such buildings in the smart grid context. By applying the possibility of having demand side management in buildings, we enable them to manage the consumption need in the end-user's side [9-10]. Meaning, that in the smart grid context, there is space and interest of calling these players to participate and be part of the management solution. These can be seen in scientific publications, such as in [11] where the aggregation of smart homes is proposed for day-ahead energy market participation, or in [12] where a home energy management system is proposed for dynamin-pricing based demand response (DR).

The integration of end-users in the smart grid can be done using DR programs [13]. These programs enable end-users to reduce the energy the energy bill. DR programs can be priceor incentive-based [14]. Depending on the DR program, the participation of end-users can be managed by the end-user or by the DR program owner. However, both approaches demand control and close to real-time monitorization in the end-user's side. And this can be achieved using IoT devices. In [15] a novel mobile app is proposed for DR clients that centralizes the household's IoT devices. Other framework for IoT integration can be seen in [16], providing a near real-time co-simulation platform using software-in-the-loop. In [17] a specialized platform oriented to support and ease the analysis of heterogeneous energy data is proposed.

In the smart grid context, an abstraction between the physical world and the player definition exists as is promoted; by using aggregators or microgrids. This paper will focus on an energy management platform for a community. Where a community is defined by a small village with public and private end-users, with energy sharing capabilities. The idea is to integrate the community the sense of union and energy sharing.

This work proposes a smart energy platform that is an improvement of an existing platform. The existing platform was developed under a Portuguese national project. The first version of the proposed platform was deployed in two communities: Alfândega da Fé, and Penela; both located in Portugal. Alfândega da Fé is a county located in the north of Portugal. With $321,95 \mathrm{~km}^{2}$ and around 5.000 habitants, it is a low-populated area. Penela is a county located in the centre of Portugal with $132.46 \mathrm{~km}^{2}$ and around 6.000 habitants. The new proposed solution brings improvements regarding its capability of integration of new IoT devices, and in the usage of artificial intelligence algorithms to produce profiling, learning and resource optimization. 
The paper describes the proposed platform's architecture and the results for its use in a community. The results for tariff decision support, consumption peak identification, and community optimization are shown in this paper.

This paper is divided in five main sections. After introduction (section I), section II will contextualize and provide a brief state of the art regarding energy communities and demand response programs. Section III presents the proposed energy management platform, and how IoT devices can be integrated. The results of the proposed platform are showed in section IV, while the main conclusions are described in section $\mathrm{V}$.

\section{COMMUNITIES AND DR PROGRAMS}

The idea of managing energy resources, namely the ones of small size, has been explored in different ways in the last years. Virtual Power Players, Virtual power Plants, Energy Islands, and Energy Communities are some of those concepts.

Looking at the share of generation benefits at local level among the community consumers is the main focus of a energy community in this paper. Energy Communities can enable energy sharing among end-users using active participation, such as demand response programs. Public and private buildings can share energy of renewable sources, such as photovoltaic and wind generation. Energy that is generated in excess can be shared among buildings.

The sharing of energy brings advantages to the entire community and to the electrical grid, eliminating the excessive use of transmission lines. The cost of energy will be lower, and people will gain a new perspective of their community and gain awareness of the benefits of renewable energy sources.

Working with communities bring new challenges that are usually not except when managing a smart grid. The energy management is done closer to end-users and they stop being numbers and start having names. And because almost all endusers know each other's, the management of energy must be explained, clarified, and introduced in the community as being part of theirs daily life.

In fact, an effective management of resources in an energy community requires an improved use of DR programs in order to induce energy consumers to change their consumption profiles according to the given price signals and/or incentives in the presence an excess of available renewables-based generation or high prices in the real-time electricity market.

\section{SMART ENERGY COMMUNITY PLATFORM}

The smart energy community platform was design and developed for communities with local energy sources, namely renewable energy sources (RES). The use of RES in endusers' buildings, for auto-consumption, brings some issues in regarding the management of energy in the building. If photovoltaic (PV) panels are used in a residential private building it will generate in the same rate as a PV panel in an office building. However, the use of such energy generation will be, usually, more efficient in the office building - where usually has its peak consumption during the day; when PV generated its peak. This is true for weekdays, but not in the weekend days where is expect a higher consumption in residential buildings during the day.

Manage the use of RES in single buildings is not straightforward because of the consumptions profiles that they have. However, looking at this problem as a community, it becomes easier to promote energy management methodologies that can reduce costs and optimize the usage of RES. This will result also in a more active participation of endusers and will raise the sense of communities. The proposed approach of this paper will use energy communities enabled by IoT devices and promote the end-user participation and energy sharing.

\section{A. Proposed architecture}

The proposed platform has the ability to list all end-users with IoT devices that are willing to actively participate in the community. The community's energy operator can see the end-users, analyse the impact of demand response programs and propose or demand action to end-users. The website platform has been developed in the last years with the main goal of using IoT devices as enables for the participation of end-users in smart grids. The approach for this participation is to use small communities. This choice follows the concept of distribution and appearance of local market and communities in the smart grid context.

Figure 1 shows the main architecture of the proposed smart energy platform. The platform will use IoT devices spread among the community; in public and also public buildings. IoT devices can be directly connected to the internet or use a gateway in the end-user side to enable its connection with the internet. The use of a gateway is not mandatory, IoT Wi-Fi-based devices can and should connect directly to the platform without the support of gateways. Only IoT devices capable of controlling the consumption of a resource and capable of measuring the resource's consumption are able to be integrated in the smart energy community platform. Otherwise, the platform lacks vital information and the device control is not effective.

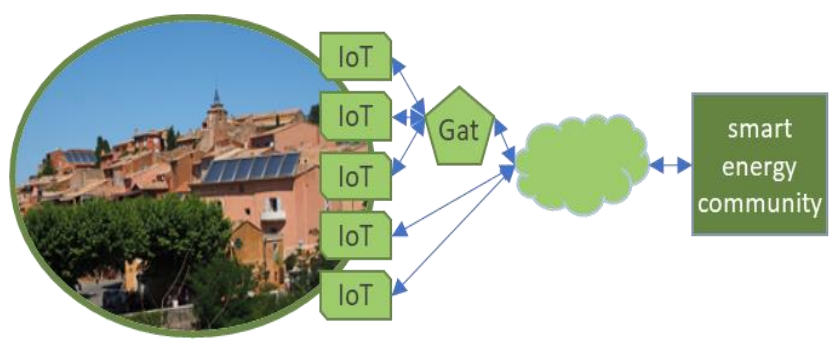

Figure 1. Proposed architecture

All devices are visible in the smart energy community platform. Each user can register IoT devices in the platform. A period after registration is used for profiling and learning. This period allows the smart energy community platform to understand when and how the IoT device is used.

After completing the profiling and learning period, the IoT device appears enabled in the platform. It is now possible to use it for active participation in the community. In the platform, IoT devices are visible inside end-users. Therefore, to register an IoT device, is needed to have the end-user already registered in the platform. The registration of endusers is free but limited to end-users with real-time metering. Without real-time metering, the platform is not able to know the total consumption and generation of the end-user and will fail to provide all its functionalities.

The platform is able to: find the best end-user energy tariff (in the point of view of reduction of cost in the end-user-side), suggest periods to end-users to decrease/increase their 
consumption, and launch demand response programs inside the community. The energy management methodologies provided in the smart energy community platform has at its centre the interests of the community, as a whole and as individual end-users.

The IoT devices, that allows the integration of end-users in the platform, are used in behalf of the overall management of the community. The platform does not provide local enduser energy management methodology. However, it can provide individual services to individual end-users, such as the analyse and decision of the best energy tariff for the user.

Figure 2 shows the graphical interface of the platform. A web-based platform is used, and the interface can be shown in a simple browser. The interface has three main section: community, end-users/clients, and simulation. The first section enables the selection of community, as well as the energy market applied in the community and the benefit sharing model that the operator wants to apply in the community. This will indicate how energy share is applied among end-users. In the section clients is where the selection of end-users is made. To prevent failures in the energy resources within the community, the platform applies a simulation using historical data.

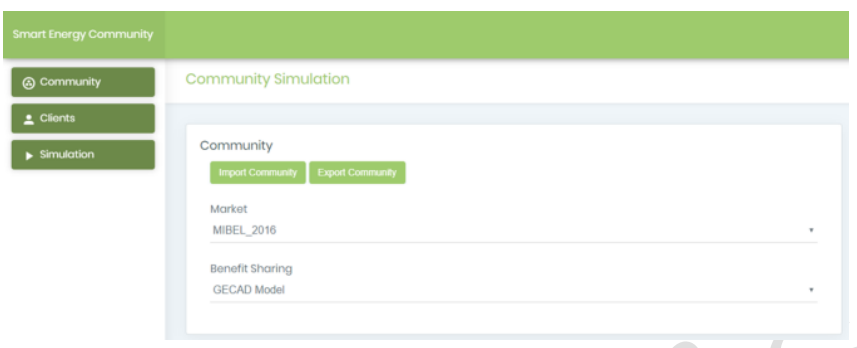

Figure 2. Smart energy community platform interface

The community's energy operator needs to analyse and validate the simulation results. After validating the data, the operator can execute the sharing model within the community. The simulation step enables the early detection of failures and also provide an easy and fast way to validate multiple models within compromising the end-users.

Figure 3 shows the simulation component to define IoT devices to be used in a end-user. For each end-user, a number of IoT devices must be specified and classified regarding its controllable resource and what is the consumption profile that will be applied in each IoT device.

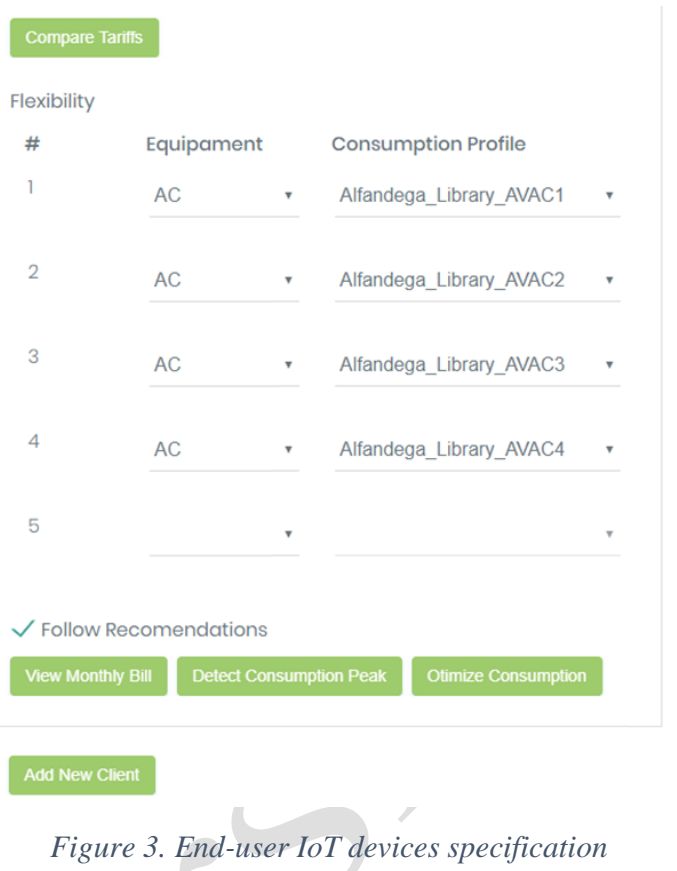

The controllable resource is the resource that is monitored and controlled by the IoT device. The choose of consumption profile enables to run multiple simulation. For instance, during the summer peak, the energy operator can simulate a winter changing the consumption profile to represent the last winter.

\section{B. IoT devices integration}

Currently, the smart energy community platform is not compliant with all IoT devices. The integration of such devices is growth in possibilities, but it is still limited when compared to the number of IoT devices capable of monitoring and controlling electrical resources.

It is not proposed any specific IoT device that the platform must use. All devices are manufactured by third parties and out of the scope of this work. The platform uses integration protocols that enables the integration of end-uses' IoT devices.

Currently, there are two protocols implemented in the smart energy community platform: message queuing telemetry transport (MQTT), and hypertext transfer protocol (HTTP). The user of the platform sees the IoT devices equal, it is not showed differentiation from MQTT nor HTTP IoT devices. The MQTT protocol is vastly used in IoT and provides a publish/subscription protocol over the TCP/IP network. Although it is not a standard in market-available product, the MQTT protocol is vastly used in IoT can be easily deployed by anyone. The smart energy community will use this protocol to subscribe energy-related values and publish control values. The platform itself, do not provide an MQTT broker, only the client is implemented in the platform. The end-user or IoT device manufacturer should provide the MQTT broker where the IoT device and the smart energy community platform will connect to.

Some IoT devices provide a HTTP application programming interface (API). This is also not a standard procedure in the IoT market, but some manufactures have open APIs. The smart energy community platform enables the use of RESTful HTTP API. IoT devices can be integrated using the uniform resource identifier (URI) for monitoring and controlling. 
In order to simply the integration of IoT devices in the platform, home automation system can be used, such as Home Assistant, OpenHab, and Domoticz. These are open-source, free to use, platforms that enable the integration of multiple IoT devices available in the current market. One of the advances behind these systems, is the availability of an API. Each of three platforms have APIs that can be used to monitor and control the IoT devices. These APIs can be used to integrate the IoT devices in the smart energy platform.

\section{RESULTS}

The results described in this section were direct results from the smart energy community. The figures showed in this section are print screens made in the web interface of the platform.

\section{A. Energy tariff and consumption peaks}

Energy tariffs are calculated per end-user using the market information applied in the community; the market can be set in the platform, as seen in Figure 2. For each end-user is possible to compare the current energy tariff with all the available tariff in the market. The results of such analyse have as base the historical consumption data of end-user.

Figure 4 shows the result tariff comparison for one enduser. In the chart is shown all the available tariff and its annual cost. Below the chart is the indication of the current end-user's tariff. Below that, in a yellow box, is shown a warning message to the community's operator indicating that a suitable tariff was found, where 274.45 EUR per year can be saved the end-user shown in Figure 4 is a public building in an estimated yearly energy cost of 7,800 EUR.

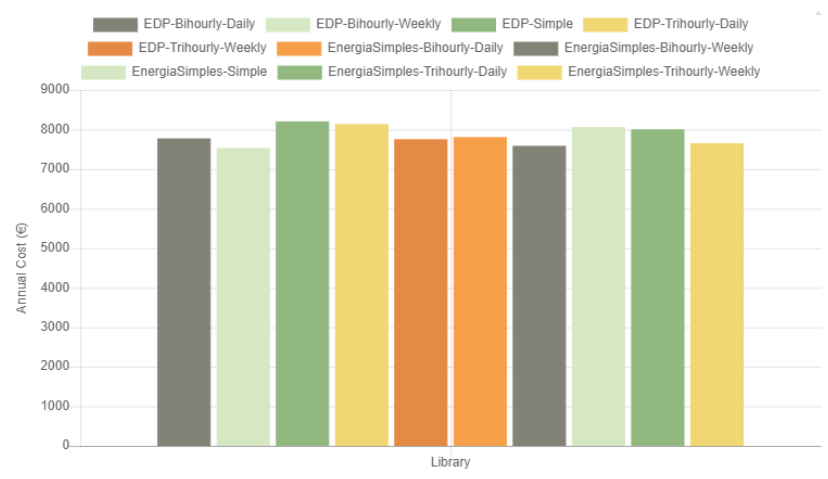

Actual Tariff: EnergiaSimples-Bihourly-Daily

The client can reduce its costs by $274.45 €$, if it joins the EDP-Bihourly-Weekly tariff.

Figure 4. End-user's tariff comparison

The proposed platform is also able to create a consumption profile per end-user. With this, it can identify periods with consumption peaks. Figure 5 shows the identification of such periods. After this identification, the community's energy operator can design and launched a demand response program to decrease the consumption in those areas. The consumption of those hours can be reduced inside the building or using a global energy reduction in the community. The platform is also able to identify peaks in the community, as in Figure 6.

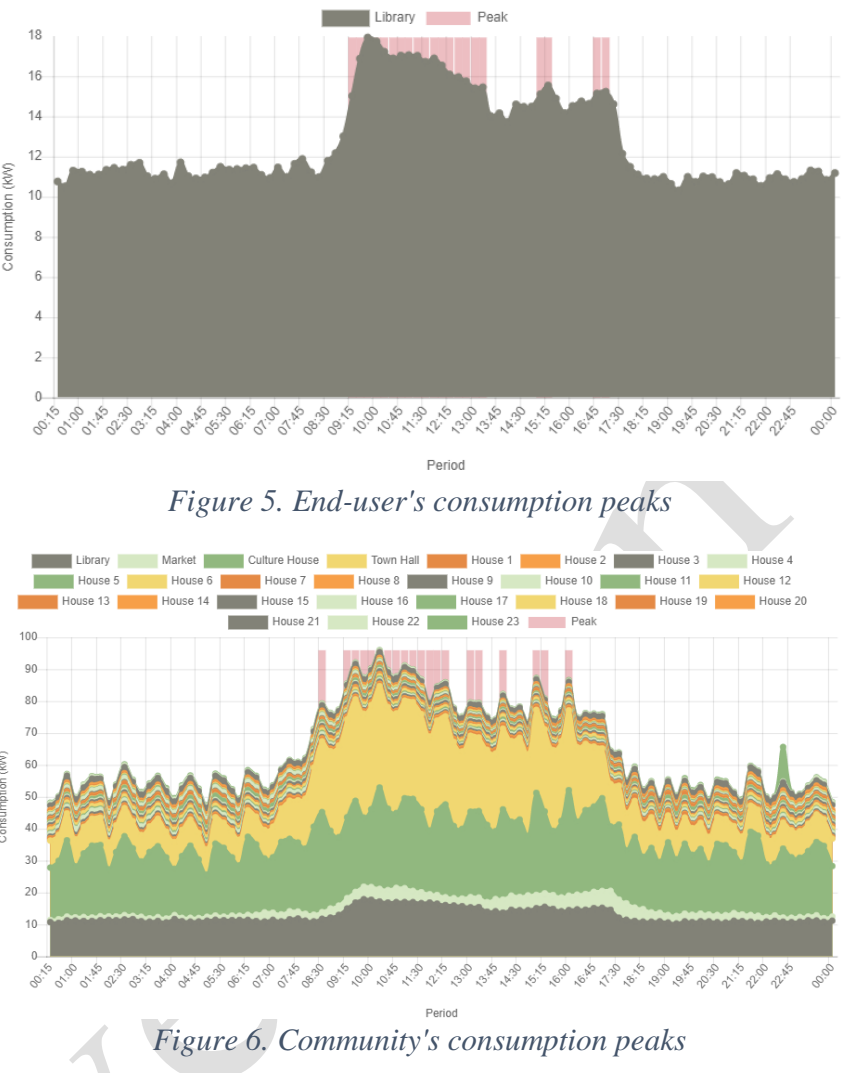

It is important to understand the community and the individual end-user. This provides more detail to the energy operator and allows the design and deployment of suitable energy management methodologies for the community as a whole.

\section{B. Energy sharing with optimization}

The energy sharing model set in the platform; according to Figure 2. Defines how the buildings' surplus energy will be distributed among the community. If, after sharing, there is still available generation, the model can specify if it will be sold to the grid and how the profit will be spread among the community. For instance, profits can be shared according to the participation of each end-user in the last months.

These results will apply energy sharing in the community. All the consumption will be optimized to decrease the consumption peaks and to use the maximum amount of energy provided by RES. The optimization will shift consumption from peak hours to out-of-peak hours. If that is not possible, a load reduction will be applied; meaning that the consumption will be reduced.

Figure 7 show the results, after optimization, for each resource that is monitored and controlled by an IoT device. All the community's IoT devices are shown in this chart. It is visible that during the day all loads, above $25 \mathrm{~kW}$ was turned off. Figure 8 shows the result of such cut. 


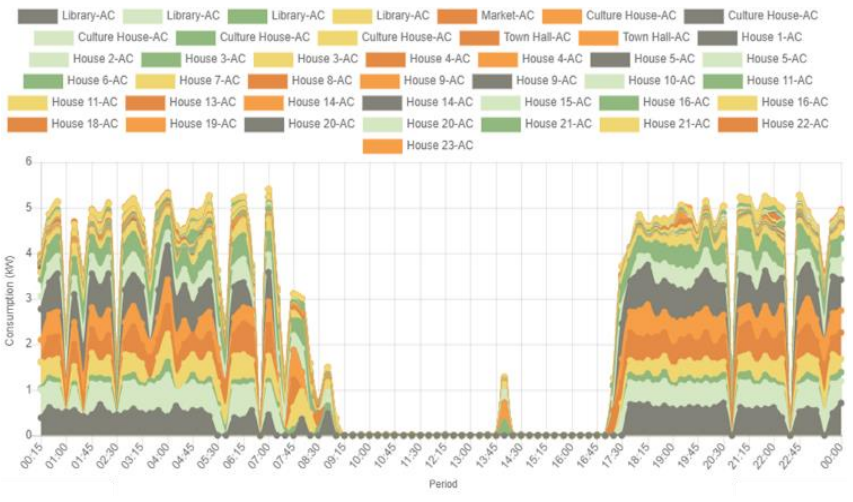

Figure 7. Consumption of resources controlled by IoT devices

Figure 8 shows the community's consumption before and after the optimization. As can be seen, the use of RES is maximized and the surplus is shared among the community, resulting in a reduction of the community's consumption during the day. The consumption peak at 22:30 is also reduced with the optimization algorithm, preventing a high energy demand in the transmission lines.

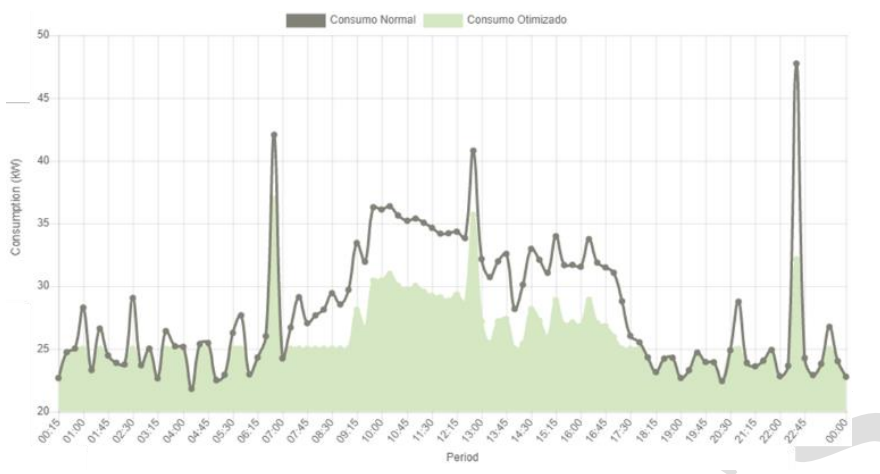

Figure 8. Community's consumption: before and after optimization

For the community energy operator, the reduction of consumption is enough. Key performance indicators (KPIs) must be draw and analysed before an energy management model is deployed in the community. The smart energy community platform supports the operator with the draw of some KPIs. These KPIs are calculated for multiple days, helping the visualization of the impact of the energy management methodology in the community. Figure 9 shows the KPIs available in the platform: consumption peak, aggregated consumption, distributed renewable generation, consumption satisfied by external generation, average energy price, and carbon dioxide $\left(\mathrm{CO}_{2}\right)$ emissions derived from the consumption.

\section{CONCLUSION}

This paper proposes a smart energy community platform that is web-based and enabled by IoT devices. The management of energy in small and medium communities bring advantages and can be a way of calling small end-users to participate in smart grids.

The proposed platform results showed the possible advantages that energy management models can have when deployed in communities. Energy sharing is also approached in this solution, involving and integrating all types of community's end-users.

The use of IoT devices is showed in this paper as enabler for the small end-users' participation. The IoT devices, aligned with the proposed platform, promote new and innovative energy management methodologies for communities. More solutions, tests and deployments of energy management systems enabled by IoT devices should be conducted in future research.

\section{ACKNOWLEDGMENT}

The present work was done and funded in the scope of the following projects: European Union's Horizon 2020 project DOMINOES (grant agreement No 771066), and UID/EEA/00760/2019 funded by FEDER Funds through COMPETE program and by National Funds through FCT. 


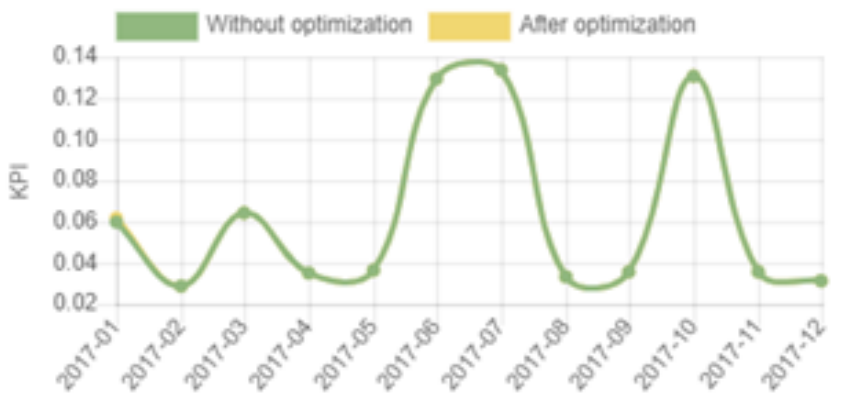

Month

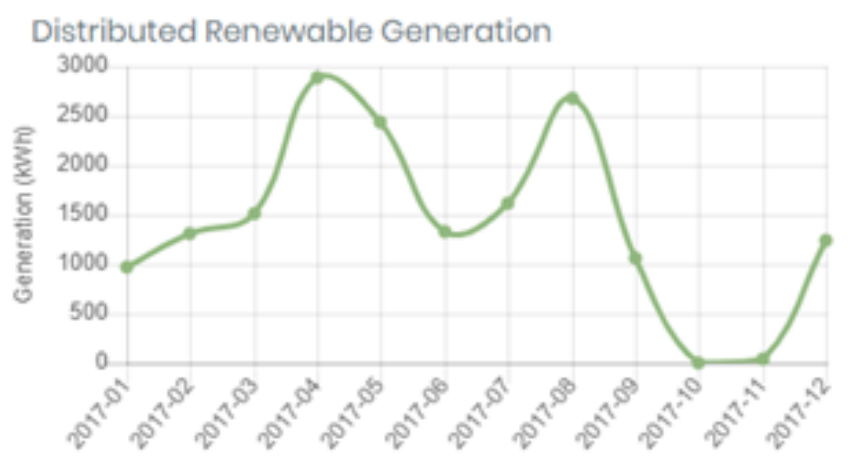

Month

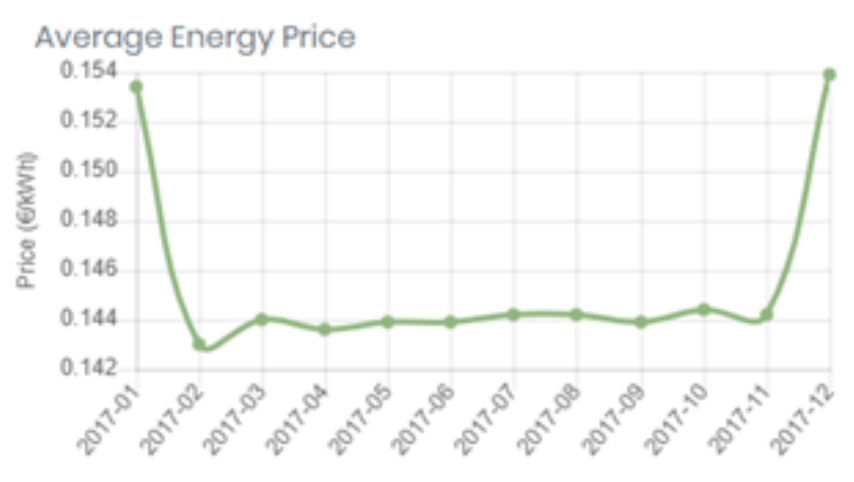

Month

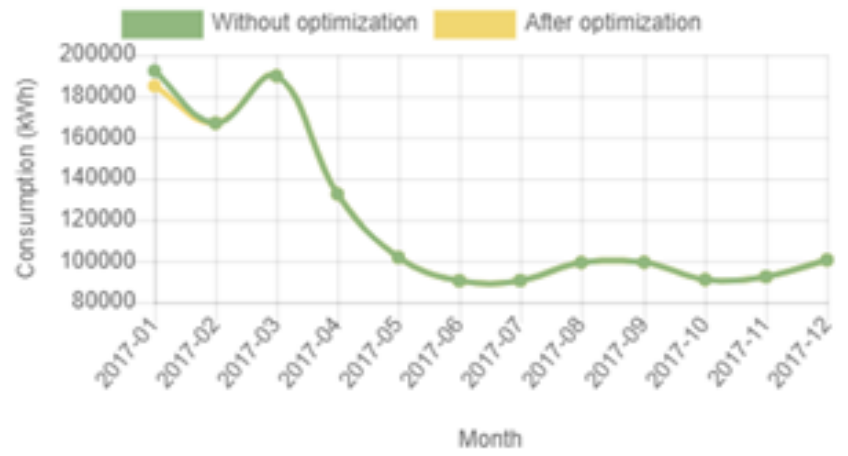

Consumption Satisfied By External Generation

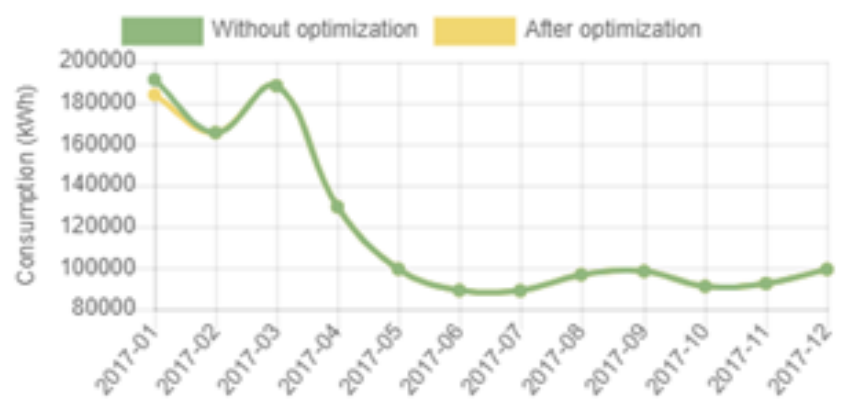

Month

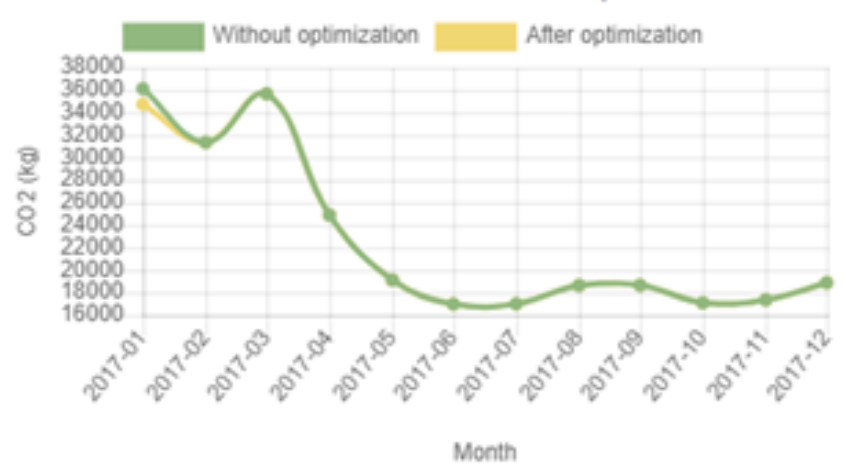

Figure 9. Community KPIs

\section{REFERENCES}

[1] Statista. Smart Home Report 2018-Control and Connectivity; Statista: Hamburg, Germany, 2018

[2] T. Alquthami, A.P.S. Meliopoulos, "Smart House Management and Control Without Customer Inconvenience", IEEE Trans. Smart Grid, 9, 2018, 2553-2562, doi:10.1109/TSG.2016.2614708

[3] Y. Liu, C. Yuen, R. Yu, Y. Zhang, S. Xie, "Queuing-Based Energy Consumption Management for Heterogeneous Residential Demands in Smart Grid", IEEE Trans. Smart Grid, 7, 2015, 1650-1659, doi:10.1109/TSG.2015.2432571.

[4] G. Galioto, N. Galioto, C. Giaconia, L. Giarré, G. Neglia,; I. Tinnirello, "Smart plugs: A low cost solution for programmable control of domestic loads", in Proceedings of the 2014 AEIT Annual Conference-From Research to Industry: The Need for a More Effective Technology Transfer (AEIT), Trieste, Italy, 18-19 September 2014; pp. 1-6, doi: 10.1109/AEIT.2014.7002015

[5] H. Morsali, S.M. Shekarabi, K. Ardekani, H. Khayamim, A. Fereidunian, M. Ghassemian,; H. Lesani, "Smart plugs for building energy management systems", in Proceedings of the Iranian Conference on Smart Grids, Tehran, Iran, 24-25 May 2012; pp. 1-5.

[6] L. Gomes, F. Sousa, Z. Vale, "An Intelligent Smart Plug with Shared Knowledge Capabilities”, Sensors, 18, 2018, 3961. doi:10.3390/s18113961
[7] T. Petrović, H. Morikawa, "Active sensing approach to electrical load classification by smart plug", in Proceedings of the 2017 IEEE Power \& Energy Society Innovative Smart Grid Technologies Conference (ISGT), Washington, DC, USA, 23-26 April 2017; pp. 1-5, doi: 10.1109/ISGT.2017.8086053

[8] Min Li, Wenbin Gu, Wei Chen, Yeshen He, Yannian Wu, Yiying Zhang, "Smart Home: Architecture, Technologies and Systems," Procedia Computer Science, vol. 131, 2018, pp. 393-400. doi: 10.1016/j.procs.2018.04.219

[9] P. Paudyal, Zhen Ni, "Smart home energy optimization with incentives compensation from inconvenience for shifting electric appliances," International Journal of Electrical Power \& Energy Systems, vol. 109, 2019, pp. 652-660. doi: 10.1016/j.ijepes.2019.02.016

[10] L. Gomes, P. Faria, H. Morais, Z. Vale and C. Ramos, "Distributed, Agent-Based Intelligent System for Demand Response Program Simulation in Smart Grids," in IEEE Intelligent Systems, vol. 29, no. 1, pp. 56-65, Jan.-Feb. 2014. doi: 10.1109/MIS.2013.2

[11] C. A. Correa-Florez, A. Michiorri, G. Kariniotakis, "Robust optimization for day-ahead market participation of smart-home aggregators," Applied Energy, vol. 229, 2018, pp. 433-445. doi: 10.1016/j.apenergy.2018.07.120

[12] O. Erdinc, N. G. Paterakis, T. D. P. Mendes, A. G. Bakirtzis and J. P. S. Catalão, "Smart Household Operation Considering Bi-Directional EV and ESS Utilization by Real-Time Pricing-Based DR," in IEEE 
Transactions on Smart Grid, vol. 6, no. 3, pp. 1281-1291, May 2015. doi: 10.1109/TSG.2014.2352650

[13] O. Erdinç, A. Taşcikaraoğlu, N. G. Paterakis and J. P. S. Catalão, "Novel Incentive Mechanism for End-Users Enrolled in DLC-Based Demand Response Programs Within Stochastic Planning Context," in IEEE Transactions on Industrial Electronics, vol. 66, no. 2, pp. 14761487, Feb. 2019. doi: 10.1109/TIE.2018.2811403

[14] A. Asadinejad, K. Tomsovic, "Optimal use of incentive and price based demand response to reduce costs and price volatility," Electric Power Systems Research, vol. 144, 2017, pp. 215-223. doi: 10.1016/j.epsr.2016.12.012

[15] D. Mashima and Wei-Peng Chen, "Residential demand response system framework leveraging IoT devices," 2016 IEEE International Conference on Smart Grid Communications (SmartGridComm), Sydney, NSW, 2016, pp. 514-520. doi: 10.1109/SmartGridComm.2016.7778813

[16] L. Barbierato et al., "A Distributed IoT Infrastructure to Test and Deploy Real-Time Demand Response in Smart Grids," in IEEE Internet of Things Journal, vol. 6, no. 1, pp. 1136-1146, Feb. 2019. doi: 10.1109/JIOT.2018.2867511

[17] F. Terroso-Saenz, A. González-Vidal, A. P. Ramallo-González, A. F. Skarmeta, "An open IoT platform for the management and analysis of energy data," Future Generation Computer Systems, vol. 92, 2019, pp. 1066-1079. doi: 10.1016/j.future.2017.08.046 